\title{
Indicadores de monitoramento da restauração na Floresta Atlântica e atributos para ecossistemas restaurados
}

\author{
Indicators for the monitoring of restoration in Atlantic Forest and attributes for restored \\ ecosystems
}

\author{
R. E. Oliveira ${ }^{1 *} ;$ V. L. Engel ${ }^{2}$ \\ ${ }^{I}$ Departamento de Desenvolvimento Rural, Universidade Federal de São Carlos, CEP 13.600-970, Araras-SP, Brasil \\ ${ }^{2}$ Departamento de Ciência Florestal, Universidade Estadual Paulista, CEP 18.610-307, Botucatu-SP, Brasil
}

*reolivei@ufscar.br

(Recebido em 13 de janeiro de 2017; aceito em 20 de dezembro de 2017)

\begin{abstract}
O objetivo deste estudo foi propor e avaliar um conjunto de indicadores, a possibilidade de seu uso efetivo e a validação por atores sociais envolvidos nos processos de avaliação da restauração da Floresta Atlântica brasileira, em diferentes fases. Com base em revisão de literatura, os indicadores foram listados e encaminhados aos atores identificados em diversos estados e em diversas instituições brasileiras. Foi solicitado a eles avaliar a importância dos indicadores listados (variando de 0 a 3 ) em diferentes estágios da restauração florestal (inicial $=2$ a 3 anos, curto prazo $=10$ anos, médio prazo $=10$ a 50 anos, longo prazo $=$ mais de 50 anos). Foi elaborada uma lista com 52 indicadores, especificamente para a avaliação de projetos de restauração florestal via plantio de mudas. Os indicadores foram categorizados em físicos e estruturais, de biodiversidade, de serviços ambientais, de processos ecológicos, econômicos e sociais. A maioria dos indicadores listados refere-se a aspectos ecológicos do processo de restauração. Sete dos nove atributos de ecossistemas restaurados propostos pela Sociedade Internacional para Restauração Ecológica são contemplados pelos indicadores listados nesta pesquisa.

Palavras-chave: Restauração florestal, Indicadores de monitoramento, Sociedade Internacional para Restauração Ecológica.
\end{abstract}

The aim of this study was to propose and evaluate set of indicators, the possibility of their effective use and their validation by stake holders in the evaluation processes of restoration in the Brazilian Atlantic Rainforest, indifferent phases. Based on literature review, indicators were listed and sent to stakeholders identified in several states and in several Brazilian institutions. They were asked to rate the importance of the indicators listed (ranging from 0 to 3 ) at different stages of forest restoration (initial $=2$ to 3 years; short term $=10$ years; medium term $=10$ to 50 years; long-term $=$ more than 50 years). A list with 52 indicators was elaborated, specifically to the evaluation of forest restoration projects using planting of seedlings in the biome. The indicators were categorized into physical and structural, biodiversity, environmental services and ecological processes, economic and social indicators. Most of the indicators listed refer to ecological aspects of the restoration process. Seven of the nine attributes for restored ecosystems proposed by the International Society for Ecological Restoration are covered by the indicators listed in this research. Keywords:Forest restoration, indicators for monitoring, Society for Ecological Restoration.

\section{INTRODUÇÃO}

Um dos grandes problemas enfrentados pelos países tropicais é o intenso processo de destruição, fragmentação e substituição de seus ecossistemas naturais. As florestas tropicais vêm sendo gradativamente reduzidas em área, tendo comprometidas sua conectividade, funcionalidade e sustentabilidade, o que está muito relacionado à destruição dos habitats florestais e ao desaparecimento de muitas de suas espécies. A possibilidade de obtenção de produtos florestais madeireiros e não madeireiros, advindos da enorme riqueza e diversidade das florestas tropicais, foi a primeira causa do processo histórico de degradação e fragmentação desses biomas, que vêm sendo amplamente utilizados ao longo da história. Posteriormente, a conversão dessas florestas de alta diversidade em áreas de agricultura e pastagem, fez com que muitas dessas áreas se tornassem ilhas em paisagens dominadas por agricultura, florestas secundárias e desenvolvimento urbano. 
As alternativas para minimizar os danos (impactos e processos de degradação) advindos da ação humana sobre as florestas tropicais incluem a criação de áreas protegidas; a implantação de áreas florestais utilizando espécies exóticas para produção de madeira e outros produtos, minimizando a pressão sobre áreas naturais; e ações de recuperação, reabilitação e restauração de ecossistemas florestais degradados. A necessidade de restauração advém de um ou mais fatores que causam alterações de grande vulto ou impactos/distúrbios que vetam ou impossibilitam a dinâmica, o funcionamento e a sustentabilidade desses ecossistemas [1,2].

Segundo a Sociedade Internacional para a Restauração Ecológica (Society for Ecological Restoration) - SER, define-se Restauração Ecológica como "o processo de auxílio ao restabelecimento de um ecossistema que foi degradado, danificado ou destruído" [3]. A restauração ecológica pode ter vários objetivos, envolver ações voltadas a diferentes tipos de ecossistemas e em diferentes escalas. As ações de restauração podem estar direcionadas para espécies em particular, para a composição da comunidade, para ecossistemas ou paisagens, e podem estar focadas nos chamados serviços ecossistêmicos [4, 5]. A avaliação e monitoramento da restauração permitem o estabelecimento de parâmetros que possibilitam saber se seus objetivos e metas foram alcançados. Essas metas devem ser mensuráveis e ter prazos mínimos estabelecidos a serem atingidos [6].

Não existe um paradigma ou contexto geral para o estabelecimento dos objetivos da restauração ecológica [7]. Entretanto, a ideia de se avaliar diferentes projetos de restauração a partir de uma base comum, centrada em um conjunto comparável de variáveis, representa uma indicação de se estar no caminho certo. Objetivos específicos bem determinados e o desenvolvimento de um conjunto de indicadores de fácil medição, que sejam claramente definidos e que possam ser monitorados, podem facilitar a definição do que seria o sucesso da restauração, possibilitando sua avaliação $[8,9]$.

A sustentabilidade dos ecossistemas florestais está associada à sua estrutura e diversidade de espécies, à ocorrência de múltiplos processos e à sua resiliência ou estabilidade. A SER definiu, em um documento traduzido para o português como "Princípios da SER International sobre Restauração Ecológica" [10], uma lista de atributos, chamados de "atributos de ecossistemas restaurados", que perfazem uma base para definir se a restauração foi alcançada. Esses atributos, aceitos internacionalmente pela comunidade científica, demonstram - em teoria - uma trajetória apropriada de desenvolvimento ecossistêmico, orientada às metas ou à referência desejada para o processo de restauração.

No caso dos ecossistemas florestais do domínio da Floresta Atlântica, a técnica mais comumente utilizada em projetos de restauração tem sido o plantio de mudas de espécies arbóreas nativas $[11,12,13]$, e vários indicadores têm sido descritos para sua avaliação [14, 15, 16, 17 , 18] sem, entretanto, haver um consenso.

Para sua efetividade, a avaliação desses projetos deve incluir a definição de objetivos de curto, médio e longo prazo no tocante a uma trajetória esperada/conhecida para a restauração, que permita definir níveis aceitáveis para determinadas variáveis, e utilizar sítios naturais como referência [19]. É importante se definir indicadores específicos para o monitoramento os quais considerem as particularidades de cada formação florestal e para áreas com condições iniciais (anteriores à restauração) distintas, assim como para as diferentes fases do processo de restauração.

Partindo desses pressupostos, este trabalho teve por objetivo construir uma lista de indicadores para monitoramento de projetos de restauração de ecossistemas florestais nativos, que (i) possam ser validados e utilizados por atores envolvidos nos processos de restauração no domínio da Floresta Atlântica, em diferentes fases do processo de restauração (ii) sejam condizentes ou estejam relacionados aos atributos de ecossistemas restaurados definidos pela Sociedade Internacional para a Restauração Ecológica. 


\section{MATERIAL E MÉTODOS}

\section{Levantamento e construção da lista de indicadores}

Foi feita ampla revisão e análise bibliográfica, focada na busca de indicadores de monitoramento da restauração, citados em fontes publicadas nas décadas de 80, 90 e 2000 (de 1980 a 2009). Esse período foi escolhido por haver consenso de que as pesquisas em restauração no Brasil se iniciaram na década de 80, e 30 anos foi então considerado tempo suficiente para se detectar tendências a partir de levantamento bibliográfico.

Na primeira etapa foi realizada busca bibliográfica utilizando a plataforma Web of Science, em que foram levantados artigos científicos que apresentassem em seus títulos o termo "restoration", em 13 periódicos (escolhidos por serem consideradas bases de referência para publicação de artigos sobre restauração ecológica e florestal): Restoration Ecology, Ecological Engineering, Conservation Biology, Biodiversity and Conservation, Journal of Applied Ecology, Forest Ecology and Management, Environmental Management, Science, Applied Vegetation Science, Biotropica, Oecologia, Landscape Ecology e Ecology. Desses, foram separados aqueles que se referiram ao uso de indicadores e/ou avaliação e monitoramento da restauração em ecossistemas florestais. Complementarmente, foi consultada a base de dados Scielo, e selecionados artigos científicos publicados, no período pretendido, abordando projetos de restauração florestal (a partir de busca com as palavras-chave "restauração", "recomposição", "revegetação", "recuperação", "reflorestamento" e "mata atlântica").

Numa segunda etapa, partindo-se do pressuposto de que, no Brasil, a grande maioria dos resultados de pesquisas em restauração para o período pretendido estaria fortemente registrada em eventos científicos e em dissertações e teses, esses foram escolhidos como alternativa para a continuidade do levantamento. Foram levantados trabalhos publicados em 18 eventos científicos realizados no Brasil (seis para cada década analisada, sendo Simpósios de Restauração de Áreas Degradadas, Congresso de Silvicultura de Espécies Nativas, Simpósios sobre Matas Ciliares, Congressos de Ecologia e Congressos de Botânica). Foram consultados os anais desses eventos, onde foram lidos os títulos de todos os resumos, resumos expandidos e trabalhos completos, entre os quais foram selecionados aqueles referentes ao tema de pesquisa. Foram pesquisadas também dissertações e teses defendidas em universidades brasileiras, cujos títulos fizeram alusão ao que se entende, atualmente, como restauração ecológica, ou, mais especificamente, restauração florestal, envolvendo fisionomias vegetais na Floresta Atlântica. Essas foram consultadas no banco de teses da CAPES (Coordenação de Aperfeiçoamento de Pessoal de Nível Superior), utilizando as mesmas palavras-chave utilizadas para consulta a base Scielo. Foram então levantados todos os trabalhos que envolveram a avaliação de áreas restauradas (ou em processo de restauração), com o uso de indicadores.

A terceira etapa envolveu busca na chamada "gray literature" (ou seja, levantamento de manuais, cartilhas e publicações técnicas no geral) e em livros e capítulos de livros que se referiram ou discutiram especificamente a restauração florestal na Floresta Atlântica brasileira, e o uso de indicadores voltados à avaliação de projetos de restauração.

A partir desse levantamento foi construída uma lista de possíveis indicadores, relacionados a aspectos diversos da restauração, contendo aqueles mencionados, sugeridos ou utilizados nos trabalhos levantados.

Essa lista foi enviada a cinco pesquisadores, atuantes na restauração e membros da Rede Brasileira de Restauração Ecológica (à época, recém-criada), pertencentes ao corpo técnico de três universidades e de duas instituições de pesquisa em quatro estados brasileiros. Foi solicitado a eles a avaliação, correção e/ou complementação da lista, corrigindo ou alterando termos e sugerindo a inclusão de indicadores que fossem considerados importantes e que não tivessem sido incluídos até então. Essas sugestões e correções foram incorporadas à versão inicial da lista de indicadores.

Os indicadores a serem avaliados foram elencados e categorizados por enfoque, como indicadores físicos e estruturais, indicadores de biodiversidade, indicadores de processos ecológicos, indicadores de serviços ambientais, indicadores econômicos e indicadores sociais. 


\section{Identificação e escolha de atores que atuam em pesquisas e ações em restauração}

Posteriormente à fase de listagem dos indicadores, foi iniciado um processo de identificação e seleção de profissionais, considerados "atores na restauração da Floresta Atlântica Brasileira", de diferentes instituições, em diferentes estados e regiões do país. Essas pessoas foram selecionadas por serem atuantes no universo da restauração, por desempenharem diferentes papéis, e por representarem diferentes setores da sociedade, a saber: (i) universidades, (ii) instituições públicas e privadas de pesquisa e desenvolvimento, (iii) órgãos governamentais - instância federal e estadual, (iv) terceiro setor e (v) prestadores de serviço ou consultores em projetos de restauração. Foram contatadas 42 pessoas, de 28 instituições, em oito estados brasileiros.

\section{Validação dos indicadores pelos atores}

Os dados utilizados nesta pesquisa podem ser descritos como procedentes de fonte primária, coletados a partir do envio de formulários auto administráveis [20]. Foi enviada uma carta aos especialistas selecionados (atores da restauração), explicando o processo e convidando-os a participar da pesquisa, e um formulário para avaliação dos indicadores. Todas as etapas no processo de avaliação, do contato ao recebimento das notas, foram realizadas via correio eletrônico, sem interação direta com os participantes, por um período de seis meses.

Solicitou-se aos participantes que avaliassem o "grau de importância" - como em Nardelli (2001) e Nardelli \& Griffith (2003) [21, 22] dos indicadores listados, pensando-se seu uso para a avaliação de projetos de restauração florestal em fitofisionomias no domínio da Floresta Atlântica, via plantio de mudas de espécies arbóreas nativas em área total.

O grau de importância de cada indicador foi sugerido numa escala crescente com notas de 0 a 3, onde: $0=0$ indicador não é importante, não deve ser considerado ou não serve para o contexto proposto; $1=\mathrm{o}$ indicador é pouco importante; $2=\mathrm{o}$ indicador é importante; $3=\mathrm{o}$ indicador é muito importante. Essa avaliação foi feita, para cada indicador, pensando-se em fases distintas (tempos diferentes) no processo de restauração, sendo: avaliação inicial (pós estabelecimento) $=$ 2 a 3 anos; curto prazo = até 10 anos; médio prazo $=10$ a 50 anos; longo prazo $=$ superior a 50 anos. Foi solicitada ainda a sugestão de outros indicadores considerados importantes pelos profissionais e aberta a possibilidade de se fazer comentários gerais sobre os indicadores listados.

Os questionários recebidos foram separados por categoria, todos os comentários realizados pelos especialistas foram anotados, e as notas fornecidas transcritas para planilhas eletrônicas.

Nesta fase da pesquisa foram verificadas as notas fornecidas pelos atores para cada um dos indicadores, para determinar se algum indicador havia sido refutado, ou seja, se algum indicador havia recebido nota zero (sendo considerado sem importância) para todas as fases propostas no processo de avaliação da restauração. Foi determinado que aqueles que recebessem nota zero, em todas as fases, seriam descartados e excluídos da lista, e que o recebimento de uma nota mínima de 1, em pelo menos uma das fases, por pelo menos um avaliador, indicaria a percepção de um grau mínimo de importância para o indicador, que seria mantido.

Após esse processo de avaliação foi obtida uma lista final de indicadores (ou aspectos a serem monitorados), considerados validados pelos atores, e que poderiam ser utilizados em processos de avaliação e monitoramento de plantios florestais na Floresta Atlântica.

\section{Paralelo entre os indicadores selecionados e os atributos da SER para ecossistemas restaurados}

Após o processo de avaliação e validação foi feita uma análise comparativa das informações, na tentativa de se estabelecer um paralelo entre os atributos (teóricos) definidos e difundidos pela Sociedade Internacional para a Restauração Ecológica e os obtidos e validados nesta pesquisa. $\mathrm{O}$ intuito foi determinar se esses se relacionavam minimamente aos atributos desejáveis para ecossistemas restaurados, e se esses atributos seriam contemplados nos processos de avaliação e monitoramento que os utilizassem. 


\section{RESULTADOS E DISCUSSÃO}

Das 42 pessoas contatadas, 17 participaram da pesquisa, manifestando sua opinião sobre os indicadores listados, a partir do método proposto, ou seja, o percentual de resposta foi de $38 \%$. Nardelli (2001) [21] considera uma taxa de 30\% de resposta em relação ao total de formulários enviados como "esperado para o tipo de entrevista e meio utilizado", ou seja, envio de questionários via correio eletrônico. Segundo Neiva (2009) [20], para um tamanho de população de 25 a 50, uma porcentagem de $20 \%$ seria considerada um tamanho de amostra medianamente rigoroso para o percentual de resposta a questionários enviados, considerado suficiente para $\mathrm{o}$ tamanho de amostra obtido.

\section{Indicadores para avaliação da restauração florestal no domínio da Floresta Atlântica}

As florestas tropicais são reconhecidas por serem altamente biodiversas e heterogêneas, por apresentarem altos níveis de endemismo, pela presença de interações planta animal complexas e especializadas e pela ocorrência de processos sucessionais associados à dinâmica de clareiras, que garantem importantes mecanismos de regeneração. A cicatrização, pela regeneração natural, de áreas abertas, ocorre em várias etapas do desenvolvimento da comunidade vegetal, como a dispersão e germinação de sementes, as diferentes fases de recrutamento e crescimento dos indivíduos e os mecanismos de reprodução das espécies. Esse ciclo é suportado por agentes polinizadores e dispersores, por mecanismos de herbivoria e competição e pela deposição de matéria e nutrientes sobre o piso florestal, entre outros processos [23, 24, 25, 26]. A presença de formas de vida animais e vegetais de grupos funcionais distintos é fundamental para o funcionamento, regeneração e dinâmica das florestas [27].

A lista construída como resultado desta pesquisa incluiu 52 itens nas seis categorias previamente definidas, sendo que a maioria ( 40 deles) refere-se a aspectos ecológicos do processo de restauração. Destes, 15 são categorizados como físicos e estruturais, 9 como de biodiversidade, 13 de processos ecológicos, 3 de serviços ecossistêmicos, 8 como econômicos e 4 referem-se a aspectos sociais da restauração (Quadro 1).

Para Hobbs \& Norton (1996) [8] o monitoramento da restauração de ecossistemas deve levar em conta sua composição, estrutura, padrão, heterogeneidade, função, interações entre espécies, dinâmica e resiliência. Ou seja, no ecossistema restaurado tem de haver um determinado conjunto de espécies que apresente padrões conhecidos de abundância e um arranjo esperado dos diferentes componentes que garanta uma estrutura vertical e distribuição horizontal condizente com as fisionomias enfocadas, e que apresente níveis mínimos de heterogeneidade e complexidade nos arranjos, na distribuição e na diversidade de componentes do sistema. Além disso, aspectos relacionados à dinâmica do ecossistema têm de estar presentes, como as interações entre espécies, processos ecológicos e resistência a distúrbios, refletindo a capacidade de auto sustentabilidade e manutenção dessas características no tempo. Esses aspectos foram contemplados nos resultados obtidos

A estrutura da vegetação está enfocada em indicadores como cobertura de copa, altura da vegetação, índice de área foliar, número de estratos, área basal, biomassa. Relativos à biodiversidade estão parâmetros de riqueza de espécies (para espécies vegetais introduzidas e não introduzidas na área, arbóreas e não arbóreas, e para espécies de fauna de vários grupos) e a presença de grupos funcionais distintos, além de espécies de diferentes grupos sucessionais e com diferentes síndromes de dispersão.

Os indicadores de processos ecológicos referem-se à ocorrência de interações (principalmente interações planta-animal) e a processos como regeneração natural (pontuada no número de espécies regenerantes e densidade de plântulas), mecanismos como banco e chuva de sementes e à deposição de matéria orgânica sobre o solo e ciclagem de nutrientes, relativos à dinâmica $\mathrm{e}$ resiliência do ecossistema em restauração.

Foi incluída também a avaliação do ambiente físico, a partir de características do solo (como estrutura, fertilidade, teor de matéria orgânica e capacidade de retenção de água) e do microclima. $\mathrm{O}$ ambiente físico muitas vezes deve ser incluído como alvo das técnicas de restauração. A recuperação gradual de características do ecossistema é planejada através de ações de restauração voltadas a ultrapassar limites (físicos e biológicos) impostos pelo processo de degradação. Em 
um ecossistema que foi degradado a partir de alterações em suas características abióticas, a restauração deve, inicialmente, concentrar esforços na remoção de fatores de degradação (como a presença de gramíneas e outras espécies exóticas invasoras, apontados aqui) e na reparação do ambiente físico e/ou químico [6, 7, 8].

Quadro 1. Indicadores numerados e elencados em suas distintas categorias, apontando aspectos a serem monitorados em processos de avaliação da restauração florestal na Floresta Atlântica brasileira

\begin{tabular}{|c|c|}
\hline $\begin{array}{c}\text { Categorias de } \\
\text { indicadores }\end{array}$ & Indicadores e aspectos a serem monitorados e avaliados \\
\hline $\begin{array}{l}\text { Indicadores físicos e } \\
\text { estruturais }\end{array}$ & $\begin{array}{l}\text { (1) Taxa de sobrevivência dos indivíduos, (2) Altura, (3) Área basal, (4) } \\
\text { Biomassa, (5) Cobertura de copa (ou da vegetação), (6) Índice de área } \\
\text { foliar, (7) Estratificação do dossel, (8) Cobertura por gramíneas } \\
\text { exóticas invasoras, (9) Presença de espécies invasoras ou indesejáveis, } \\
\text { (10) Microclima, (11) Estrutura do solo, (12) Fertilidade do solo, (13) } \\
\text { Capacidade de retenção de água, (14) Teor de matéria orgânica, (15) } \\
\text { Relação C/N na serapilheira. }\end{array}$ \\
\hline $\begin{array}{l}\text { Indicadores de } \\
\text { biodiversidade }\end{array}$ & $\begin{array}{l}\text { (16) Riqueza de espécies vegetais nativas, (17) Presença e diversidade } \\
\text { de outras formas de vida vegetais, (18) Presença de aves, (19) Presença } \\
\text { de mamíferos, (20) Presença de insetos, (21) Presença de mesofauna do } \\
\text { solo, (22) Presença de microfauna do solo, (23) Presença de espécies de } \\
\text { diferentes grupos sucessionais, (24) Presença de espécies com } \\
\text { diferentes síndromes de dispersão. } \\
\text { (25) Número de espécies regenerantes (nativas) diferentes das }\end{array}$ \\
\hline $\begin{array}{c}\text { Indicadores de } \\
\text { processos ecológicos }\end{array}$ & $\begin{array}{l}\text { introduzidas inicialmente na área, (26) Densidade de plântulas nativas, } \\
\text { (27) Produção de folhedo ou serapilheira, (28) Banco de sementes como } \\
\text { mecanismo de regeneração, (29) Chuva de sementes como mecanismo } \\
\text { de regeneração, (30) Ocorrência de herbivoria, (31) Presença de } \\
\text { micorrizas, (32) Ocorrência de polinização, (33) Ocorrência de } \\
\text { dispersão de sementes pela fauna, (34) Parasitismo, (35) Fluxo gênico, } \\
\text { (36) Taxas de cruzamento, (37) Frutificação }\end{array}$ \\
\hline $\begin{array}{c}\text { Indicadores de } \\
\text { serviços ambientais }\end{array}$ & $\begin{array}{l}\text { (38) Estoques de carbono, (39) Presença de erosão e sedimentação, (40) } \\
\text { Regulação do regime hídrico } \\
\text { 41) Custo de elaboração do projeto, (42) Custo de implantação, (43) }\end{array}$ \\
\hline $\begin{array}{l}\text { Indicadores } \\
\text { econômicos }\end{array}$ & $\begin{array}{c}\text { Custo de manutenção inicial (combate a invasoras e pragas), (44) Custo } \\
\text { de manutenção contínua da área (aceiros, cercas, outros), (45) Taxas de } \\
\text { incremento e produtividade, (46) Renda líquida, (47) Número de } \\
\text { produtos madeireiros e não madeireiros obtidos, (48) Aptidão de } \\
\text { benefício econômico por serviços ambientais }\end{array}$ \\
\hline $\begin{array}{l}\text { Indicadores } \\
\text { sociais }\end{array}$ & $\begin{array}{l}\text { (49) Grau de aceitação da ação de restauração pela comunidade local, } \\
\text { (50) Participação da comunidade local na elaboração do projeto, (51) } \\
\text { Participação da comunidade local na implantação do projeto, (52) Valor } \\
\text { etnobotânico das espécies presentes }\end{array}$ \\
\hline
\end{tabular}

Também foram listados indicadores de serviços ecossistêmicos, como regulação de regime hídrico, controle de erosão e sedimentação e estoque de carbono. É importante destacar que, historicamente, ações de restauração na paisagem rural dos estados com cobertura original pertencente à Floresta Atlântica enfocaram prioritariamente as áreas de preservação permanente, associadas à conservação de recursos hídricos.

De acordo com Aronson et al (2009) [28] existe ainda clara necessidade de se definir e avaliar resultados socioeconômicos dos projetos de restauração ecológica, e as numerosas ligações entre restauração, desenvolvimento econômico e bem-estar social devem ser ressaltadas e explicitadas sempre que possível.Os indicadores listados incluem a avaliação de benefícios econômicos, associados à geração de renda advinda de produtos florestais madeireiros e não madeireiros obtidos das espécies implantadas. 
A obrigatoriedade de restauração das reservas legais em $20 \%$ da área das propriedades rurais $[29,30]$ pode ser um aspecto interessante a ser enfocado e avaliado, por incluir ainda a possibilidade de obtenção de renda pelo pagamento por serviços ambientais e remuneração por serviços ecossistêmicos, temas muito discutidos atualmente, no que se refere à construção de políticas públicas voltadas à restauração.

Os indicadores econômicos referem-se também a custos no processo de restauração. Os altos custos relatados para a restauração florestal no Brasil são considerados um grande limitante [31, 13], o que provavelmente explica a ocorrência desses indicadores (custos de elaboração, implantação e manutenção inicial e contínua) para avaliação de projetos e ações.

Para Geist \& Galatowitsch (1999) [32] cabe a integração de elementos naturais e humanos no planejamento e implementação da restauração, a partir do desenvolvimento de relações benéficas mútuas. Os progressos na restauração acontecem à medida em que se desenvolvem e ocorrem benefícios mútuos entre componentes ambientais e humanos. Contribuições das populações humanas ocorrem quando as populações se envolvem na restauração (recebendo benefícios por isso), e as contribuições ambientais na medida em que mais ecossistemas degradados vão sendo restaurados. À medida que as necessidades humanas vão sendo atendidas (recebendo bens e serviços ambientais), o envolvimento e empenho na restauração - ou na participação e aceitação da necessidade desse processo - aumentam.

Os indicadores sociais elencados estão muito relacionados à aceitação e participação da sociedade no planejamento e na realização de ações de restauração. Incluem também o valor etnobotânico das espécies, ou seja, relacionam-se também à preferência das populações locais, envolvidas nos processos de restauração, quanto à escolha das espécies utilizadas.

Como já apontado, em teoria, indicadores devem abordar aspectos que possam ser facilmente mensurados. Devem também ser replicáveis e passíveis de comparação, a fim de possibilitar a avaliação dos mesmos aspectos em diferentes localidades e projetos de restauração (considerando-se os mesmos ecossistemas de referência e fitofisionomias base para avaliação da trajetória da restauração). Dentre os aspectos elencados aqui, vários podem ser considerados indicadores mensuráveis (como, por exemplo, altura, área basal, cobertura de copa ou por gramíneas, riqueza de espécies, densidade de espécies regenerantes, número de estratos presentes, etc.). Outros, entretanto, podem ser considerados atributos que devem ter seus indicadores melhor definidos ou caracterizados (como presença ou ausência de determinados processos, formas de vida ou grupos funcionais, e características relacionadas ao microclima e estrutura do solo), e aspectos mais gerais, como aqueles relacionados aos serviços ecossistêmicos, ou a aspectos sociais/culturais (participação da comunidade ou aceitação pelas comunidades envolvidas dos projetos de restauração avaliados).

Como o levantamento realizado se baseou na literatura, todos os aspectos apontados como "indicadores" foram considerados e inseridos na análise, mas talvez nem todos aqueles aqui listados possam ser assim considerados e, para cada um deles, cabe melhor avaliação.

Embora alguns indicadores não tenham sido avaliados, por alguns atores, em alguma das etapas propostas (alguns optaram pela resposta "não saberia avaliar" em alguns casos), todos os indicadores foram validados, ou seja, nenhum deles recebeu nota zero em todas as etapas do processo de restauração (fase inicial, curto, médio e longo prazo) então, aparentemente, todos são passíveis de utilização na avaliação de projetos. Cabe ressaltar que não foram incluídos novos indicadores pelos avaliadores consultados.

\section{Indicadores para Restauração Florestal e relação com os atributos de ecossistemas restaurados}

A SER considera prioritariamente aspectos ecológicos, referentes à estrutura e diversidade, à presença de espécies nativas e de grupos funcionais e a um ambiente físico adequado como relevantes a serem observados, quando descreve os nove atributos desejáveis aos ecossistemas restaurados (Quadro 2). Esses atributos referem-se a (i) diversidade e estrutura da comunidade similar a locais de referência; (ii) presença de espécies nativas no maior grau possível; (iii) presença dos grupos funcionais considerados necessários à estabilidade do sistema; (iv) ambiente físico capaz de suportar populações reprodutivas; (v) funcionamento normal; (vi) integração com 
a paisagem; (vii) eliminação de ameaças potenciais; (viii) resiliência a distúrbios naturais; (xix) auto sustentabilidade. Destes, somente dois não foram contemplados pelos indicadores aqui listados (os atributos 6 e 7), já que nenhum deles está relacionado à avaliação da restauração no tocante à integração com a paisagem ou a fluxos e/ou outras interações entre a área em processo de restauração com a matriz ecológica na qual está inserida. Também não há alusão à avaliação ou monitoramento de ameaças à saúde e integridade do ecossistema ou de distúrbios em geral, a partir dos indicadores propostos.

Pode-se considerar que os demais atributos foram contemplados pela lista de indicadores construída nesta pesquisa e podem ser avaliados ou monitorados a partir dos aspectos elencados e validados pelos atores entrevistados.

Por exemplo, estão presentes a composição de espécies característica do ecossistema, a predominância de espécies nativas e a representação de grupos funcionais como indicativos de estabilidade. A avaliação do ambiente físico, como base para a sustentação dessas espécies é enfocada a partir de características de clima e solo, e atributos relativos à saúde do ecossistema estão presentes nas categorias de indicadores que pretendem mensurar o funcionamento de seus processos e aspectos dinâmicos (a partir dos indicadores de processos ecológicos e de serviços ecossistêmicos).

A resiliência e autossuficiência do ecossistema em restauração são abordadas por indicadores de processos ecológicos e serviços, relativos à sua capacidade de sustentação, resistência a novos distúrbios e sustentabilidade no tempo.

\section{A seleção de indicadores ideais para avaliação e monitoramento de projetos de restauração florestal}

Para avaliação do processo de restauração, é necessária a indicação de um ecossistema de referência para comparação, o que nem sempre é uma tarefa fácil. Durigan (2011) [33] aponta que a regra para escolha dessa referência pode ser a de que esse ecossistema deve representar "o melhor resultado possível para a realidade sócio- econômica e a condição de degradação em que se encontra a área em restauração".Esse melhor resultado possível tem relação direta com a trajetória natural desses ecossistemas, ou seja, existe uma condição prévia esperada, para o momento em que se encontra o ecossistema, em uma escala temporal, considerando-se os processos sucessionais ou de regeneração e padrões de referência em termos de sua estrutura, composição e funcionamento. Nas palavras de Suganuma \& Durigan (2014) [34], dentro de uma mesma região ecológica, a restauração via plantio de mudas de espécies arbóreas (alvo desta pesquisa) resulta em uma trajetória padrão, para a maioria dos atributos de estrutura, riqueza e guildas, que deve orientar o estabelecimento de metas para a restauração. Essas metas vão auxiliar a escolha dos melhores indicadores para o monitoramento.

Não existe uma fórmula pré-estabelecida, cada sítio degradado possui sua história de degradação e está sujeito a um conjunto de características ecossistêmicas, merecendo assim estratégias específicas de restauração [35]. Para escolha dos indicadores o raciocínio é o mesmo, nem todo indicador é possível de ser aplicado e/ou utilizado em todas as situações, o que se aplica também aos indicadores apontados aqui como resultado desta pesquisa.

Podem ser elencados alguns critérios para a escolha e proposição dos melhores indicadores para uma determinada situação. Algumas características são apontadas como ideais para indicadores ecológicos [36, 37, 38, 33], das quais podem ser destacadas: facilidade de identificação do indicador, facilidade de medição, sensibilidade aos impactos ao sistema e resposta previsível a esses impactos, obtenção de respostas que permitam a comparação com outros sistemas e a previsão sobre os efeitos de ações e práticas de manejo que venham a ser aplicadas.

No Estado de São Paulo, por exemplo, o protocolo de monitoramento de projetos de restauração ecológica definiu somente três indicadores a serem aferidos e seus valores de referência, para diferentes tipologias vegetacionais de ocorrência no estado. Esses indicadores são cobertura do solo com vegetação nativa, densidade de indivíduos nativos regenerantes e número de espécies nativas regenerantes [39, 40]. Esses três indicadores referem-se a características de estrutura, composição de espécies e funcionamento (processos ecológicos como sucessão 
secundária e regeneração), que indicam potencial de sustentabilidade para o ecossistema em avaliação. Em se tratando das fitofisionomias alvo deste protocolo, o monitoramento no tempo dessas variáveis permitirá acompanhar avanços ou retrocessos na trajetória da restauração.

Quadro 2. Paralelo entre os atributos de ecossistemas restaurados definidos pela Society for Ecological Restoration (SER, 2004) e os indicadores listados (identificados por número, conforme o Quadro 1), bem como as categorias onde se inserem ( $F=$ físicos e estruturais; $B=$ biodiversidade; $P=$ processos ecológicos; $S e=$ serviços ambientais; $E=$ econômicos; $S=$ sociais; $N$. c. = não contemplado).

\begin{tabular}{|c|c|c|c|}
\hline Atributos & $\begin{array}{c}\text { Descrição dos atributos } \\
\text { desejáveis a ecossistemas } \\
\text { restaurados } \\
\end{array}$ & Indicadores & Categorias \\
\hline 1 & $\begin{array}{c}\text { O E.R. contém uma } \\
\text { composição de espécies } \\
\text { característica que ocorre no } \\
\text { ecossistema de referência que } \\
\text { mantêm a estrutura da } \\
\text { comunidade }\end{array}$ & $3,5,7,16$ a 26 & F, B \\
\hline 2 & $\begin{array}{c}\text { O E.R. consiste, em sua } \\
\text { "maior extensão praticável”, } \\
\text { de espécies nativas }\end{array}$ & 16 a 26 & B \\
\hline 3 & $\begin{array}{l}\text { O E.R. tem representados } \\
\text { todos os grupos funcionais } \\
\text { necessários à sua } \\
\text { estabilidade, ou os que não } \\
\text { estão presentes têm potencial } \\
\text { de colonização }\end{array}$ & 18 a 24,31 & $\mathrm{~B}, \mathrm{P}$ \\
\hline 4 & $\begin{array}{l}\text { O E.R. apresenta um } \\
\text { ambiente físico capaz de } \\
\text { sustentar populações. } \\
\text { reprodutivas das espécies } \\
\text { necessárias para manter a } \\
\text { estabilidade em sua trajetória }\end{array}$ & $5,7,8,10$ a 15 & $\mathrm{~F}$ \\
\hline 5 & $\begin{array}{l}\text { O E.R. possui um } \\
\text { funcionamento normal para o } \\
\text { estágio de desenvolvimento } \\
\text { em que se encontra }\end{array}$ & $\begin{array}{c}7 \text { a } 15,23,25 \text { a } 37,38 \\
\text { a } 40\end{array}$ & $\mathrm{P}, \mathrm{Se}$ \\
\hline 6 & $\begin{array}{c}\text { O E.R. está integrado à } \\
\text { paisagem ou a uma matriz } \\
\text { ecológica, com a qual } \\
\text { interage (fluxos físicos e } \\
\text { biológicos) }\end{array}$ & N. c. & N. c. \\
\hline 7 & $\begin{array}{l}\text { Não existem ameaças à saúde } \\
\text { e integridade do E.R., ou } \\
\text { essas foram reduzidas ao } \\
\text { máximo }\end{array}$ & N. c. & N. c. \\
\hline 8 & $\begin{array}{c}\text { O E.R. éresiliente o } \\
\text { suficiente para reagir a } \\
\text { situações de stress ou } \\
\text { distúrbios }\end{array}$ & $\begin{array}{c}7 \text { a } 15,23,25 \text { a } 37,38 \\
\text { a } 40\end{array}$ & $F, B, P, S e$ \\
\hline 9 & $\begin{array}{l}\text { O E.R. é autossuficiente no } \\
\text { mesmo grau do ecossistema } \\
\text { referência, e tem potencial de } \\
\text { persistir indefinidamente sob } \\
\text { diferentes condições } \\
\text { ambientais }\end{array}$ & $\begin{array}{c}7 \text { a } 15,23,25 \text { a } 37 \text { e } \\
38 \text { a } 40\end{array}$ & $F, B, P, S e$ \\
\hline
\end{tabular}


Cabe chamar a atenção, finalmente, ao fato de que existem aspectos que não somente os ecológicos, a serem considerados quando se pensa a avaliação do processo de restauração. Para Higgs (1997) [41] a restauração não terá sucesso incluindo apenas aspectos biológicos e/ou ecológicos, e deverá considerar aspectos históricos, sociais, culturais, políticos, estéticos e morais.

A própria SER, que inicialmente considera somente atributos ecológicos para se mensurar o quão restaurado se pode considerar um determinado ecossistema, indica que outros atributos têm relevância e deveriam ser incluídos, desde que identificados como objetivos iniciais de um projeto de restauração, como a produção de bens e serviços voltados ao benefício social. Vallauri et al (2005) [42] apontam que a avaliação da restauração deve enfocar (e alcançar) os objetivos iniciais propostos, ou ao menos, redefini-los, e a base para avaliação e monitoramento deve levar em conta, além da integridade ecológica e benefícios ambientais, o bem estar e a qualidade de vida das populações humanas.

Para Hobbs (2016) [43] as motivações, quantidade de recursos disponíveis e a escala de restauração é que vão determinar tipo e intensidade das intervenções consideradas apropriadas em cada projeto. Em cada caso, há todo um conjunto de valores a ser considerado, pensando-se sistemas em diferentes estados. Nesse ínterim, o autor destaca a controvérsia referente a ecossistemas já muito modificados (os "novel ecosystems") e as melhores formas de se pensar sua restauração, bem como a avaliação desses processos.

Mansourian \& Vallauri (2014) [44] destacam que a restauração de paisagens florestais é abordagem cada vez mais relevante para a temática da conservação ambiental, pois aborda vários desafios atuais, como mudanças climáticas, segurança alimentar, disponibilidade de água doce e mitigação de desastres naturais. Entretanto, a consideram mal compreendida e subestimada, e pouco considerada quando da avaliação de projetos de restauração. Para os autores, as necessidades socioeconômicas dos atores locais não são suficientemente reconhecidas ou abordadas em programas de restauração em larga escala, e essa omissão pode afetar negativamente seu sucesso. Atributos socioeconômicos são apontados como lacuna também por Wortley et al (2013) [45], para quem a quantificação de serviços ecossistêmicos e outros resultados socioeconômicos é essencial para se compreender os reais benefícios e os custos completos da restauração ecológica.

Esses apontamentos podem subsidiar a tomada de decisão sobre quais dos indicadores (ou aspectos a monitorar) aqui listados devem ser prontamente descartados e quais realmente podem ser utilizados para monitoramento da trajetória da restauração em ecossistemas florestais no domínio da Floresta Atlântica.

\section{CONCLUSÃO}

Nesta pesquisa foram levantados 52 indicadores para o monitoramento da restauração florestal, validados por atores sociais atuantes na restauração florestal no domínio da Floresta Atlântica, a fim de enriquecer a discussão sobre os atributos de uma boa restauração ecológica, pensando-se no contexto ecológico, econômico, social e cultural, e considerando-se a realidade das áreas a serem restauradas, em processo de restauração ou consideradas restauradas.

Apontam linhas gerais sobre o conjunto de características normalmente utilizado ou citado para o monitoramento da restauração (via plantio de mudas de espécies arbóreas), tendo por base a trajetória pretendida para ecossistemas florestais, e indicam aspectos que podem ser considerados e monitorados, no tempo.

É importante frisar, entretanto, que para cada projeto ou ação, a avaliação e/ou monitoramento deve enfocar seus objetivos iniciais e metas definidas para a restauração, e são esses objetivos que irão determinar o que deve - ou não - ser monitorado. Irão determinar quais os aspectos a serem monitorados, quais os indicadores a serem utilizados, quando e como, tendo em vista o contexto (espacial/ecológico e sócio/econômico/cultural) onde se insere a ação de restauração, a visão e experiência dos agentes restauradores, e sua capacidade de utilizar e avaliar os indicadores propostos. 


\section{AGRADECIMENTOS}

Este trabalho é parte de uma pesquisa intitulada "O estado da arte da Ecologia da Restauração e sua relação com a restauração de ecossistemas florestais no Bioma Mata Atlântica", financiada pela Fundação de Amparo à Pesquisa do Estado de São Paulo (Processo FAPESP 2008/531837), à qual agradecemos pelo suporte financeiro. Cabe ressaltar que as opiniões, hipóteses e conclusões ou recomendações expressas neste material são de responsabilidade de suas autoras e não necessariamente refletem a visão da FAPESP.

Agradecemos também aos profissionais que participaram da pesquisa para avaliação dos indicadores de restauração e àqueles que auxiliaram complementarmente, na construção e correção da lista final de indicadores.

\section{REFERÊNCIAS BIBLIOGRÁFICAS}

1. Lamb D, Erskine PD, Parrotta J. Restoration of degraded forest landscapes. Science. 2005 Dez;310:1628-1632, doi:10.1126/science.1111773.

2. Gann GD, Lamb D. Ecological restoration: A mean of conserving biodiversity and sustaining livelihoods (version 1.1). Society for Ecological Restoration International, Tucson, Arizona, USA and IUCN, Gland, Switzerland. 2006. Disponível em: www.ser.org.

3. Society for Ecological Restoration. The SER international primer on ecological restoration. Tucson, 2004. Disponível em: <http://www.ser.org/content/ecological_restoration_primer.asp>. Acesso em 10 out. 2007.

4. Mansourian S. Overview of forest restoration strategies and terms. New York (USA): Springer, WWF; 2005. Chapter, Forest restoration in landscape: beyond planting trees; p. 8-13, doi:10.1007/0-38729112-1_2.

5. Palmer MA. Ecological theory and restoration ecology. Washington (USA): Island Press, 2006. Chapter, Foundations of restoration ecology; p. 1-10.

6. Aronson J, Durigan G, Brancalion PHS. Conceitos e definições correlatos à ciência e à prática da Restauração Ecológica. IF Série Registros. 2011 Ago;44:1-38.

7. Ehrenfeld JG. Defining the limits of restoration: the need for realistical goals. Restoration Ecology. 2000 Mar;8(1):2-9.

8. Hobbs R, Norton DA. Towards a conceptual framework for restoration ecology. Restoration Ecology. 1996 Jun;4(2):93-110.

9. Ruiz-Jaen MC, Aide TM. Restoration success: how is it being measured? Restoration Ecology. 2005 Set;13(3):569-577.

10. Society for ecological restoration. 2011. Princípios da SER International sobre a restauração ecológica. http://c.ymcdn.com/sites/www.ser.org/resource/resmgr/custompages/publications/SER_Primer/serprimer-portuguese.pdf.

11. Rodrigues RR, Lima RAF, Gandolfi S, Nave AG. On the restoration of high diversity forests: 30 year experience in the Brazilian Atlantic Forest. Biological Conservation. 2009 142:1242-1251, doi:10.1016/j.biocon.2008.12.008

12. Brancalion, PHS. Instrumentos legais podem contribuir para a restauração de florestas tropicais biodiversas. Revista Árvore. 2010 34(3):455-470.

13. Rodrigues RR, Gandolfi S, Nave AG, Aronson J, Barreto TE, Vidal CY, Brancalion PHS. Large-scale ecological restoration of high-diversity tropical forests in SE Brazil. Forest Ecology and Management. 2011 Mai;261(10):1605-1613, doi:10.1016/j.foreco.2010.07.005.

14. Rodrigues RR. Restauração de florestas tropicais: indicadores de avaliação e monitoramento vegetal. Piracicaba: ESALQ, 1999. (Manuscrito)

15. Kageyama PY, Gandara FB. Recuperação de áreas ciliares. São Paulo (Brasil): Edusp/FAPESP, 2001. Matas ciliares: conservação e recuperação; p. 249-269.

16. Silva AN. Recuperação Florestal: da muda à floresta. São Paulo (Brasil): Secretaria do Meio Ambiente do Estado de São Paulo; 2004. 111 p.

17. Moraes LFD. Indicadores da restauração de áreas degradadas na Reserva Biológica de Poço das Antas, RJ. [Tese]. Rio de Janeiro (RJ): Universidade Federal Rural do Rio de Janeiro; 2005. 107 p.

18. Rodrigues RR, Brancalion PHS, Isernhagen I. Pacto pela restauração da Mata Atlântica: referencial dos conceitos e ações de restauração florestal. São Paulo (SP): ESALQ, LERF; Instituto BioAtlântica; 2009. $264 \mathrm{p}$.

19. Engel VL. Indicadores de sucesso na restauração ecológica. In: Congresso Iberoamericano e do Caribe sobre Restauração Ecológica, 2009, Curitiba. Anais... Curitiba: SOBRADE, 2009. CD-ROM. 
20. Neiva A. As áreas de preservação permanente no Brasil: a percepção de especialistas. [Dissertação] Viçosa (MG): Universidade Federal de Viçosa; 2009. 119 p.

21. Nardelli AMB. Sistemas de certificação e visão de sustentabilidade no setor florestal brasileiro. [Tese] Viçosa (MG): Universidade Federal de Viçosa; 2001. 119 p.

22. Nardelli AMB, Griffith JJ. Mapeamento conceitual da visão da sustentabilidade de diferentes atores no setor florestal brasileiro. Revista Árvore. 2003 27(2):241-256.

23. Bierregaard RO, Lovejoy TE, Kapos V, Santos, AA, Hutchings, RW. The Biological Dynamics of Tropical Rainforest Fragments. BioScience. 1992 Dez;42(11):859- 866.

24. Lima RAF, Moura LC. Canopy Gap Colonization in the Atlantic Montane Rain Forest. Brazilian Archives of Biology and Technology. 2006 Nov;49(6):953-965.

25. Dent DH, De Walt SJ, Denslow JS. Secondary forests of central Panama increase in similarity tooldgrowth forest over time in shade tolerance but not species composition. Journal of Vegetation Science. 2012 Oct;24(3):530-542, doi:10.1111/j.1654-1103.2012.01482.x

26. Arihafa A, Mack AL. Treefall Gap Dynamics in a Tropical Rain Forest in Papua New Guinea. Pacific Science. 2013 67(1):47-58, doi:http://dx.doi.org/10.2984/67.1.4

27. Denslow JS. 1996 Functional group diversity and responses to disturbance. Berlin: Springer-Verlag; 1996. Biodiversity and Ecosystem Processes in Tropical Forests; p.127-151.

28. Aronson J, Blignaut JM, Milton SJ, Le Maitre D, Esler KJ, Limouzin A, Fontaine C, de Wit MP, Mugido W, Prinsloo P, Van der Elst L, Lederer N. 2010. Are socioeconomic benefits of restoration adequately quantified? A meta-analysis of recent papers (2000-2008) in Restoration Ecology and 12 other scientific journals. Restoration Ecology. 2010 Mar;18(2):143-154, doi:10.1111/j.1526100X.2009.00638.X

29. Soares-Filho B, Rajão R, Macedo M, Carneiro A, Costa W, Coe M, Rodrigues H, Alencar A. Cracking Brazil's Forest Code. Science. 2014 Abr;344:363-364, doi:10.1126/science.1246663.

30. Brancalion PHS, Garcia LC, Loyola R, Rodrigues RR, Pillar V, Lewinsohn TM. Análise crítica da Lei de Proteção da Vegetação Nativa (2012), que substituiu o antigo Código Florestal: atualizações e ações em curso. Brazilian Journal of Natural Conservation. 2016 14:e1-e16, doi:http://dx.doi.org/10.1016/j.ncon.2016.03.004.

31. Oliveira RE, Souza AM, Rodrigues CL, Romero ML. Aspectos da recuperação e uso de florestas em propriedades e paisagens rurais no Estado de São Paulo. São Paulo (SP): Imprensa Oficial; 2008. Recuperação Florestal: um olhar social, p. 45-78.

32. Geist C, Galatowitsch SM. Reciprocal model for meeting ecological and human needs in restoration projects. Conservation Biology. 1999 13(5):970-979.

33. Durigan G. O uso de indicadores para monitoramento das áreas em recuperação. Cadernos da Mata Ciliar. 2011, 4:11-21.

34. Suganuma MS, Durigan G. Indicators of restoration success in riparian tropical forests using multiple reference ecosystems. Restoration Ecology. 2014 23(3):238-251, doi:10.1111/rec.12168.

35. Moraes LFD, Campello EFC, Franco AA. Restauração florestal: do diagnóstico de degradação ao uso de indicadores ecológicos para o monitoramento das ações. Oecologia Australis. 2010 Jun;14(2):437451, doi:10.4257/oeco.2010.1402.07.

36. Andreasen JK, O'neill RV, Noss R, Slosser NC. Considerations for the development of a terrestrial index of ecological integrity. Ecological Indicators. 2001 Ago;1:21-35, doi:10.1016/S1470$160 \mathrm{X}(01) 00007-3$.

37. Dale VH, Beyeler SC. Changes in the development and use of ecological indicators. Ecological Indicators. Aug 2001;1:3-10, doi:https://doi.org/10.1016/S1470-160X(01)00003-6.

38. Manoliadis OG. Development of ecological indicators: a methodological framework using compromise programming. Ecological Indicators. 2002 Nov;2:169-176, doi:https://doi.org/10.1016/S1470-160X(02)00040-7.

39. São Paulo, Secretaria de Estado do Meio Ambiente. Coordenadoria de Biodiversidade e Recursos Naturais. Portaria 01/2015. Estabelece o Protocolo de Monitoramento de Projetos de Restauração Ecológica. 2015, $10 \mathrm{p}$.

40. Chaves RB, Durigan G, Brancalion PHS, Aronson, J. On the need of legal frameworks for assessing restoration projects success: new perspectives from São Paulo state (Brazil). Restoration Ecology. 2015 Nov;23(6):754-759, doi:10.1111/rec.12267

41. Higgs E. What is good ecological restoration? Conservation Biology. 1997 11(2):338-348.

42. Vallauri D. Monitoring and evaluating forest restoration success. New York (NY): Springer; WWF, 2005. Forest restoration in landscape: beyond planting trees, p. 151-156.

43. Hobbs R. Degraded or just different? Perceptions and value judgments in restoration decisions. Restoration Ecology. 2016 Mar;24(2):153-158, doi:10.1111/rec.12336. 
44. Mansourian S, Vallauri D. Restoring Forest Landscapes: important lessons learnt. Environmental Management. 2014 53:241-251, doi:10.1007/s00267-013-0213-7.

45. Wortley L, Hero JM, Howes M. Evaluating ecological restoration success: a review of the literature. Restoration Ecology. 2013 Set;21(5):537-543, doi:10.1111/rec.12028. 